\title{
On interpolative $F$-contractions with shrink map
}

\author{
Monairah Alansari ${ }^{1}$ and Muhammad Usman $\mathrm{Ali}^{2^{*}}$
}

"Correspondence:

muh_usman_ali@yahoo.com;

musman.ali@ciit-attock.edu.pk ${ }^{2}$ Department of Mathematics, COMSATS University Islamabad, Attock Campus, Attock, Pakistan Full list of author information is available at the end of the article

\begin{abstract}
In this article, we introduce two notions of interpolative F-contractions with shrink map and $F$-contractions with shrink map. We also study the existence of $E$-fixed points by using these notations on a metric space endowed with a binary relation. As an application and consequence of the main results, we also get some other interesting results like a common fixed point result, an $E$-fixed point result on a metric space equipped with graph, and an existence theorem for a solution of integral equations.

MSC: $47 \mathrm{H} 10 ; 54 \mathrm{H} 25$

Keywords: E-fixed points; Interpolative Kannan contraction; Interpolative F-contraction with shrink map
\end{abstract}

\section{Introduction and preliminaries}

The concept of interpolative Kannan contraction mapping was derived by Karapinar [1] to redefine the famous Kannan contraction mapping in the following way: A mapping $V:\left(K, d_{K}\right) \rightarrow\left(K, d_{K}\right)$ is an interpolative Kannan contraction [1] if

$$
d_{K}(V k, V l) \leq \gamma\left[d_{K}(k, V k)\right]^{\tau_{1}}\left[d_{K}(l, V l)\right]^{1-\tau_{1}}
$$

for all $k, l \in K$ with $k \neq V k$, where $\gamma \in[0,1)$ and $\tau_{1} \in(0,1)$.

After that, Karapınar, Agarwal, and Aydi [2] refined the above inequality as

$$
d_{K}(V k, V l) \leq \gamma\left[d_{K}(k, V k)\right]^{\tau_{1}}\left[d_{K}(l, V l)\right]^{1-\tau_{1}}
$$

for all $k, l \in K \backslash \operatorname{Fix}(V)$, where $\gamma \in[0,1), \tau_{1} \in(0,1)$ and $\operatorname{Fix}(V)=\{k \in K: V k=k\}$.

This concept of interpolative contraction provides a new dimension of study whether existing contraction inequalities can be redefined in this way or not. This concept of Karapinar [1] provoked research in this field, and within a short duration we have seen many new studies related to this topic. For example, Gaba and Karapinar [3] modified the interpolative Kannan contraction by using different exponential values instead of $1-\tau_{1}$, which were independent of $\tau_{1}$. Karapinar et al. [4] redefined the Hardy-Rogers type contraction by the interpolative Hardy-Rogers type contraction. Reich-Rus-Ćirić type contractions were extended to interpolative Reich-Rus-Ćirić type contractions by Karapınar et

(c) The Author(s) 2021. This article is licensed under a Creative Commons Attribution 4.0 International License, which permits use, sharing, adaptation, distribution and reproduction in any medium or format, as long as you give appropriate credit to the original author(s) and the source, provide a link to the Creative Commons licence, and indicate if changes were made. The images or other third party material in this article are included in the article's Creative Commons licence, unless indicated otherwise in a credit line to the material. If material is not included in the article's Creative Commons licence and your intended use is not permitted by statutory regulation or exceeds the permitted use, you will need to obtain permission directly from the copyright holder. To view a copy of this licence, visit http://creativecommons.org/licenses/by/4.0/. 
al. [2] and Aydi et al. [5]. Karapınar [6] also studied interpolative contractions based on simulation functions. Mohammadi et al. [7] studied interpolative Ćirić-Reich-Rus type contractions in the sense of Wardowski [8]. Altun and Tasdemir [9] presented the study of best proximity points using interpolative proximal contraction inequalities. Along with the aforementioned studies, many other interesting studies on fixed point theory are available in [10-23]; they help readers to verify the existence of fixed points for self-mappings and best proximity points for nonself mappings.

Jleli et al. [23] introduced the concept of $E$-fixed point (also called $\phi$-fixed point), which states that, for maps $V: K \rightarrow K$ and $E: K \rightarrow[0, \infty)$, a point $k \in K$ is called $E$-fixed point of $V: K \rightarrow K$ if $V(k)=k$ and $E(k)=0$, and proved the existence of such points by using a single inequality involving both maps $V$ and $E$. It is important to note that Jleli et al. [23] used the lower semicontinuity of $E$. This use of the lower semicontinuity of $E$ by Jleli et al. [23] arises the question whether the condition of lower semicontinuity of $E$ can be left and some other technique be adopted. In this research article, we try to investigate the results by applying the following conditions to the aforementioned study by Jleli et al. [23].

(I) If the condition of lower semicontinuity is not applied to $E$.

(II) And if two different inequalities are used for $V$ and $E$ instead of a single inequality as used by Jleli et al. [23].

As an application and consequence of the main results, we get some other interesting results like a common fixed point result, an $E$-fixed point result on a metric space equipped with graph, and an existence theorem for a solution of integral equations.

In the next section, we use the family of functions given by Wardowski [8]. $\Omega$ is a set of functions $F:(0, \infty) \rightarrow \mathbb{R}$ with the following properties:

(F1) $k_{1}<k_{2} \Leftrightarrow F\left(k_{1}\right)<F\left(k_{2}\right) \forall k_{1}, k_{2} \in(0, \infty)$;

(F2) for each $\left\{k_{n}: k_{n}>0\right\}$, we get $\lim _{n \rightarrow \infty} k_{n}=0$ if and only if $\lim _{n \rightarrow \infty} F\left(k_{n}\right)=-\infty$;

(F3) there exists $t \in(0,1)$ with $\lim _{k \rightarrow 0^{+}}(k)^{t} F(k)=0$.

\section{Main results}

In this section we assume that $\left(K, d_{K}\right)$ is a metric space equipped with a binary relation $R_{B}$ and $V: K \rightarrow K, E: K \rightarrow[0, \infty)$ are two maps. The set of all natural numbers is represented by $\mathbb{N}$, and the set of all whole numbers is represented by $\mathbb{W}$.

Definition 2.1 A map $V$ is called interpolative $F$-contraction with $E$ shrink if the following inequalities exist:

$$
\Lambda+F\left(d_{K}(V k, V z)\right) \leq \tau_{1} F\left(d_{K}(k, z)\right)+\tau_{2} F\left(d_{K}(k, V k)\right)+\tau_{3} F\left(d_{K}(z, V z)\right)
$$

for each $k, z \in K$ with $k R_{B} z$ and

$$
\min \left\{d_{K}(V k, V z), d_{K}(k, V k), d_{K}(z, V z)\right\}>0,
$$

where $F \in \Omega, \Lambda>0$ and $\tau_{1}, \tau_{2}, \tau_{3} \in(0,1)$ with $\tau_{1}+\tau_{2}+\tau_{3}=1$;

for each $k \in K$, we have

$$
E(V k) \leq \lambda E(k)
$$

where $\lambda \in[0,1)$. 
Note that $\left(K, d_{K}\right)$ equipped with a binary relation $R_{B}$ is called complete in the sense of $R_{B}$ if, for each sequence $\left\{k_{n}\right\}$ in $K$ with $\lim _{n, m \rightarrow \infty} d_{K}\left(k_{n}, k_{m}\right)=0$ and $k_{n} R_{B} k_{n+1} \forall n \in \mathbb{N}$, we have $k_{b} \in K$ with $\lim _{n \rightarrow \infty} d_{K}\left(k_{n}, k_{b}\right)=0$ and $k_{n} R_{B} k_{b} \forall n \geq n_{0}$ (for some $n_{0} \in \mathbb{N}$ ).

Now we are in a position to state and prove the first result.

Theorem 2.2 Consider $V$ to be an interpolative F-contraction with $E$ shrink on a metric space $\left(K, d_{K}\right)$ equipped with a binary relation $R_{B}$. Also, consider that

(a) there exist $k_{0} \in K$ and $k_{1}=V k_{0}$ such that $k_{0} R_{B} k_{1}$;

(b) if $k, l \in K$ with $k R_{B} l$, then $V k R_{B} V l$, that is, $V$ is $R_{B}$-preserving;

(c) $\left(K, d_{K}\right)$ is complete in the sense of $R_{B}$;

(d) either $V$ is continuous or $F$ is continuous.

Then there exists $a$ point $b \in K$ with $V b=b$ and $E b=0$.

Proof The existence of axiom (a) implies $k_{0} \in K$ and $k_{1}=V k_{0}$ such that $k_{0} R_{B} k_{1}$. Then, by repeated application of axiom (b), we say that $V^{n} k_{0} R_{B} V^{n+1} k_{0} \forall n \in \mathbb{N}$. Consider $k_{n}=V^{n} k_{0}$ $\forall n \in \mathbb{W}$, then $k_{n} R_{B} k_{n+1} \forall n \in \mathbb{W}$. Suppose that

$$
\min \left\{d_{K}\left(V k_{n_{0}}, V k_{n_{0}+1}\right), d_{K}\left(k_{n_{0}}, V k_{n_{0}}\right), d_{K}\left(k_{n_{0}+1}, V k_{n_{0}+1}\right)\right\}=0 \quad \text { for some } n_{0} \in \mathbb{W} \text {, }
$$

then either $k_{n_{0}}$ or $k_{n_{0}+1}$ is a fixed point of $V$. Say, it is $k_{n_{0}}$; by (2.2), we obtain $E\left(k_{n_{0}}\right)=$ $E\left(V k_{n_{0}}\right) \leq \lambda E\left(k_{n_{0}}\right)$, that is, $E\left(k_{n_{0}}\right)=0$. Hence, the conclusion is proved. Now assume that

$$
\min \left\{d_{K}\left(V k_{n}, V k_{n+1}\right), d_{K}\left(k_{n}, V k_{n}\right), d_{K}\left(k_{n+1}, V k_{n+1}\right)\right\}>0 \quad \forall n \in \mathbb{W},
$$

then (2.1) implies

$$
\begin{aligned}
\Lambda+F\left(d_{K}\left(V k_{n}, V k_{n+1}\right)\right) \leq & \tau_{1} F\left(d_{K}\left(k_{n}, k_{n+1}\right)\right)+\tau_{2} F\left(d_{K}\left(k_{n}, V k_{n}\right)\right) \\
& +\tau_{3} F\left(d_{K}\left(k_{n+1}, V k_{n+1}\right)\right) \quad \forall n \in \mathbb{W} .
\end{aligned}
$$

That is,

$$
\begin{aligned}
\Lambda+F\left(d_{K}\left(k_{n+1}, k_{n+2}\right)\right) \leq & \tau_{1} F\left(d_{K}\left(k_{n}, k_{n+1}\right)\right)+\tau_{2} F\left(d_{K}\left(k_{n}, k_{n+1}\right)\right) \\
& +\tau_{3} F\left(d_{K}\left(k_{n+1}, k_{n+2}\right)\right) \quad \forall n \in \mathbb{W} .
\end{aligned}
$$

Here, we claim $d_{K}\left(k_{n+1}, k_{n+2}\right)<d_{K}\left(k_{n}, k_{n+1}\right)$ for all $n \in \mathbb{W}$. Assume in contrast that there is $n_{*} \in W$ with $d_{K}\left(k_{n_{*}+1}, k_{n_{*}+2}\right) \geq d_{K}\left(k_{n_{*}}, k_{n_{*}+1}\right)$. By keeping this fact and increasing natural of $F,(2.4)$ becomes

$$
\begin{aligned}
\Lambda+F\left(d_{K}\left(k_{n_{*}+1}, k_{n_{*}+2}\right)\right) \leq & \tau_{1} F\left(d_{K}\left(k_{n_{*}}, k_{n_{*}+1}\right)\right)+\tau_{2} F\left(d_{K}\left(k_{n_{*}}, k_{n_{*}+1}\right)\right) \\
& +\tau_{3} F\left(d_{K}\left(k_{n_{*}+1}, k_{n_{*}+2}\right)\right) \\
\leq & \tau_{1} F\left(d_{K}\left(k_{n_{*}+1}, k_{n_{*}+2}\right)\right)+\tau_{2} F\left(d_{K}\left(k_{n_{*}+1}, k_{n_{*}+2}\right)\right) \\
& +\tau_{3} F\left(d_{K}\left(k_{n_{*}+1}, k_{n_{*}+2}\right)\right) \\
= & F\left(d_{K}\left(k_{n_{*}+1}, k_{n_{*}+2}\right)\right)
\end{aligned}
$$


which is not possible. Hence, the claim is true, that is, $d_{K}\left(k_{n+1}, k_{n+2}\right)<d_{K}\left(k_{n}, k_{n+1}\right)$ for all $n \in \mathbb{W}$. Hence, by (2.4), we obtain

$$
\begin{aligned}
\Lambda+F\left(d_{K}\left(k_{n+1}, k_{n+2}\right)\right) \leq & \tau_{1} F\left(d_{K}\left(k_{n}, k_{n+1}\right)\right)+\tau_{2} F\left(d_{K}\left(k_{n}, k_{n+1}\right)\right) \\
& +\tau_{3} F\left(d_{K}\left(k_{n+1}, k_{n+2}\right)\right) \\
\leq & F\left(d_{K}\left(k_{n}, k_{n+1}\right)\right) \quad \forall n \in \mathbb{W} .
\end{aligned}
$$

This gives

$$
F\left(d_{K}\left(k_{n+1}, k_{n+2}\right)\right) \leq F\left(d_{K}\left(k_{0}, k_{1}\right)\right)-(n+1) \Lambda \quad \forall n \in \mathbb{W} .
$$

From (2.7), we get $\lim _{n \rightarrow \infty} F\left(d_{K}\left(k_{n+1}, k_{n+2}\right)\right)=-\infty$. This implies $\lim _{n \rightarrow \infty} d_{K}\left(k_{n+1}, k_{n+2}\right)=0$ due to (F2). Setting $d_{K}\left(k_{n+1}, k_{n+2}\right)=\xi_{n+1}$, thus, $\lim _{n \rightarrow \infty} \xi_{n+1}=0$. Condition (F3) ensures that there is $t \in(0,1)$ such that $\lim _{n \rightarrow \infty} \xi_{n+1}^{t} F\left(\xi_{n+1}\right)=0$. From (2.7), we obtain

$$
\xi_{n+1}^{t} F\left(\xi_{n+1}\right)-\xi_{n+1}^{t} F\left(\xi_{0}\right) \leq-\xi_{n+1}^{t}(n+1) \Lambda<0 \quad \forall n \in \mathbb{W} .
$$

Then $\lim _{n \rightarrow \infty} \xi_{n+1}^{t}(n+1)=0$. Thus, there exists $N_{*} \in \mathbb{N}$ with $\xi_{n+1} \leq \frac{1}{(n+1)^{\frac{1}{t}}} \forall n \geq N_{*}$. For every $m, n \in \mathbb{N}$ with $m>n$, we obtain

$$
\begin{aligned}
d_{K}\left(k_{n}, k_{m}\right) & \leq d_{K}\left(k_{n}, k_{n+1}\right)+d_{K}\left(k_{n+1}, k_{n+2}\right)+\cdots+d_{K}\left(k_{m-1}, k_{m}\right) \\
& \leq \sum_{j=n}^{\infty} \frac{1}{j^{\frac{1}{t}}} .
\end{aligned}
$$

Thus, $\lim _{n, m \rightarrow \infty} d_{K}\left(k_{n}, k_{m}\right)=0$, since $\sum_{j=1}^{\infty} \frac{1}{j^{\frac{1}{t}}}$ is convergent. Hence, we get a Cauchy $\left\{k_{n}\right\}$ with $k_{n} R_{B} k_{n+1} \forall n \in \mathbb{W}$. As we have already assumed in the statement of the theorem that $\left(K, d_{K}\right)$ is complete in the sense of $R_{B}$, thus, there is $k_{*} \in K$ with $k_{n} \rightarrow k_{*}$ and $k_{n} R_{B} k_{*}$ for each $n>m_{0}$ (for some $m_{0}$ ).

Suppose that $V$ is continuous with respect to $d_{K}$, and we know $\lim _{n \rightarrow \infty} d_{K}\left(k_{n}, k_{*}\right)=0$, thus, $0=\lim _{n \rightarrow \infty} d_{K}\left(V k_{n}, V k_{*}\right)=\lim _{n \rightarrow \infty} d_{K}\left(k_{n+1}, V k_{*}\right)=d_{K}\left(k_{*}, V k_{*}\right)$. Hence, we conclude that $V k_{*}=k_{*}$.

Suppose that $F$ is continuous. Here, we claim $V k_{*}=k_{*}$. Suppose, in contrast, that it is wrong. Then we conclude that

$$
\min \left\{d_{K}\left(V k_{n}, V k_{*}\right), d_{K}\left(k_{n}, V k_{n}\right), d_{K}\left(k_{*}, V k_{*}\right)\right\}>0 \quad \forall n \geq m_{a} \in \mathbb{N} .
$$

As $k_{n} R_{B} k_{*}$ for each $n>m_{0}$, by (2.1), we get

$$
\begin{aligned}
\Lambda+F\left(d_{K}\left(V k_{n}, V k_{*}\right)\right) \leq & \tau_{1} F\left(d_{K}\left(k_{n}, k_{*}\right)\right)+\tau_{2} F\left(d_{K}\left(k_{n}, V k_{n}\right)\right) \\
& +\tau_{3} F\left(d_{K}\left(k_{*}, V k_{*}\right)\right) \quad \forall n>m_{b}=\max \left\{m_{a}, m_{0}\right\} .
\end{aligned}
$$

As $\lim _{n \rightarrow \infty} d_{K}\left(k_{n}, k_{*}\right)=0, \lim _{n \rightarrow \infty} d_{K}\left(k_{n}, k_{n+1}\right)=0$, and $d_{K}\left(k_{*}, V k_{*}\right) \neq 0$, thus, there is $N_{0} \in$ $\mathbb{N}$ with

$$
\max \left\{d_{K}\left(k_{n}, k_{*}\right), d_{K}\left(k_{n}, k_{n+1}\right), d_{K}\left(k_{*}, V k_{*}\right)\right\}=d_{K}\left(k_{*}, V k_{*}\right) \quad \forall n \geq N_{0}
$$


Using it in (2.9), we obtain

$$
\Lambda+F\left(d_{K}\left(k_{n+1}, V k_{*}\right)\right) \leq\left(\tau_{1}+\tau_{2}+\tau_{3}\right) F\left(d_{K}\left(k_{*}, V k_{*}\right)\right) \quad \forall n>\max \left\{m_{b}, N_{0}\right\}
$$

Assuming that $n \rightarrow \infty$ and using the continuity of $F$ in (2.10), we obtain

$$
\Lambda+F\left(d_{K}\left(k_{*}, V k_{*}\right)\right) \leq\left(\tau_{1}+\tau_{2}+\tau_{3}\right) F\left(d_{K}\left(k_{*}, V k_{*}\right)\right)
$$

which is not possible for $d_{K}\left(k_{*}, V k_{*}\right) \neq 0$, that is, a contradiction to our assumption. Hence, the claim is true, that is, $k_{*}=V k_{*}$. Consider $k=k_{*}$ in (2.2), we get $E\left(V k_{*}\right) \leq \lambda E\left(k_{*}\right)$, that is, $(1-\lambda) E\left(k_{*}\right) \leq 0$. Hence, $E\left(k_{*}\right)=0$.

Now we present the notion of $F$-contraction with shrink map in the following way.

Definition 2.3 A map $V$ is called $F$-contraction with $E$ shrink if the following inequalities exist:

$$
\Lambda+F\left(d_{K}(V k, V z)\right) \leq F\left(\tau_{1} d_{K}(k, z)+\tau_{2} d_{K}(k, V k)+\tau_{3} d_{K}(z, V z)\right)
$$

for each $k, z \in K$ with $k R_{B} z$ and $V k \neq V z$, where $F \in \Omega, \Lambda>0$ and $\tau_{1}, \tau_{2}, \tau_{3} \in[0,1]$ with $\tau_{1}+\tau_{2}+\tau_{3}=1$

for each $k \in K$, we have

$$
E(V k) \leq \lambda E(k)
$$

where $\lambda \in[0,1)$

Now we shall discuss the second result of this section.

Theorem 2.4 Consider $V$ to be an F-contraction with $E$ shrink on a metric space $\left(K, d_{K}\right)$ with a binary relation $R_{B}$. Also, consider that

(a) there exist $k_{0} \in K$ and $k_{1}=V k_{0}$ such that $k_{0} R_{B} k_{1}$;

(b) if $k, l \in K$ with $k R_{B} l$, then $V k R_{B} V l$, that is, $V$ is $R_{B}$-preserving;

(c) $\left(K, d_{K}\right)$ is complete in the sense of $R_{B}$;

(d) either $V$ is continuous or $F$ is continuous.

Then there exists a point $b \in K$ with $V b=b$ and $E b=0$.

Proof Axiom (a) says that $k_{0} \in K$ and $k_{1}=V k_{0}$ satisfy $k_{0} R_{B} k_{1}$. From axiom (b), we conclude that $V^{n} k_{0} R_{B} V^{n+1} k_{0} \forall n \in \mathbb{N}$. Letting $k_{n}=V^{n} k_{0} \forall n \in \mathbb{W}$, we get $k_{n} R_{B} k_{n+1} \forall n \in \mathbb{W}$. Suppose that there is some $n_{0} \in \mathbb{W}$ with $k_{n_{0}}=k_{n_{0}+1}=V k_{n_{0}}$, then $k_{n_{0}}$ is a fixed point of $V$. From (2.12), we obtain $E\left(k_{n_{0}}\right)=E\left(V k_{n_{0}}\right) \leq \lambda E\left(k_{n_{0}}\right)$, that is, $E\left(k_{n_{0}}\right)=0$. Hence, the conclusion is proved. Now assume that $d_{K}\left(V k_{n}, V k_{n+1}\right)>0 \forall n \in \mathbb{W}$, then (2.11) implies

$$
\begin{aligned}
\Lambda+F\left(d_{K}\left(V k_{n}, V k_{n+1}\right)\right) \leq & F\left(\tau_{1} d_{K}\left(k_{n}, k_{n+1}\right)+\tau_{2} d_{K}\left(k_{n}, V k_{n}\right)\right. \\
& \left.+\tau_{3} d_{K}\left(k_{n+1}, V k_{n+1}\right)\right) \quad \forall n \in \mathbb{W} .
\end{aligned}
$$


That is,

$$
\begin{aligned}
\Lambda+F\left(d_{K}\left(k_{n+1}, k_{n+2}\right)\right) \leq & F\left(\tau_{1} d_{K}\left(k_{n}, k_{n+1}\right)+\tau_{2} d_{K}\left(k_{n}, k_{n+1}\right)\right. \\
& \left.+\tau_{3} d_{K}\left(k_{n+1}, k_{n+2}\right)\right) \quad \forall n \in \mathbb{W} .
\end{aligned}
$$

Here, we claim $d_{K}\left(k_{n+1}, k_{n+2}\right)<d_{K}\left(k_{n}, k_{n+1}\right) \forall n \in \mathbb{W}$. Suppose, in contrast, that there is $n_{*} \in W$ with $d_{K}\left(k_{n_{*}+1}, k_{n_{*}+2}\right) \geq d_{K}\left(k_{n_{*}}, k_{n_{*}+1}\right)$. By this fact and increasing natural of $F$, (2.14) becomes

$$
\begin{aligned}
\Lambda+F\left(d_{K}\left(k_{n_{*}+1}, k_{n_{*}+2}\right)\right) \leq & F\left(\tau_{1} d_{K}\left(k_{n_{*}}, k_{n_{*}+1}\right)+\tau_{2} d_{K}\left(k_{n_{*}}, k_{n_{*}+1}\right)\right. \\
& \left.+\tau_{3} d_{K}\left(k_{n_{*}+1}, k_{n_{*}+2}\right)\right) \\
\leq & F\left(\tau_{1} d_{K}\left(k_{n_{*}+1}, k_{n_{*}+2}\right)+\tau_{2} d_{K}\left(k_{n_{*}+1}, k_{n_{*}+2}\right)\right. \\
& \left.+\tau_{3} d_{K}\left(k_{n_{*}+1}, k_{n_{*}+2}\right)\right) \\
= & F\left(d_{K}\left(k_{n_{*}+1}, k_{n_{*}+2}\right)\right),
\end{aligned}
$$

which is not possible. Hence, the claim is valid, that is, $d_{K}\left(k_{n+1}, k_{n+2}\right)<d_{K}\left(k_{n}, k_{n+1}\right) \forall n \in \mathbb{W}$. Hence, by (2.14), we obtain

$$
\begin{aligned}
\Lambda+F\left(d_{K}\left(k_{n+1}, k_{n+2}\right)\right) \leq & F\left(\tau_{1} d_{K}\left(k_{n}, k_{n+1}\right)+\tau_{2} d_{K}\left(k_{n}, k_{n+1}\right)\right. \\
& \left.+\tau_{3} d_{K}\left(k_{n+1}, k_{n+2}\right)\right) \\
\leq & F\left(d_{K}\left(k_{n}, k_{n+1}\right)\right) \quad \forall n \in \mathbb{W} .
\end{aligned}
$$

This implies

$$
F\left(d_{K}\left(k_{n+1}, k_{n+2}\right)\right) \leq F\left(d_{K}\left(k_{0}, k_{1}\right)\right)-(n+1) \Lambda \quad \forall n \in \mathbb{W} .
$$

By (2.17), we get $\lim _{n \rightarrow \infty} F\left(d_{K}\left(k_{n+1}, k_{n+2}\right)\right)=-\infty$. This implies $\lim _{n \rightarrow \infty} d_{K}\left(k_{n+1}, k_{n+2}\right)=0$ from (F2). Let $d_{K}\left(k_{n+1}, k_{n+2}\right)=\xi_{n+1}$, thus, $\lim _{n \rightarrow \infty} \xi_{n+1}=0$. (F3) ensures that there is $t \in$ $(0,1)$ with $\lim _{n \rightarrow \infty} \xi_{n+1}^{t} F\left(\xi_{n+1}\right)=0$. By $(2.17)$, we get

$$
\xi_{n+1}^{t} F\left(\xi_{n+1}\right)-\xi_{n+1}^{t} F\left(\xi_{0}\right) \leq-\xi_{n+1}^{t}(n+1) \Lambda<0 \quad \forall n \in \mathbb{W} .
$$

Thus, $\lim _{n \rightarrow \infty} \xi_{n+1}^{t}(n+1)=0$. Then there exists $N_{*} \in \mathbb{N}$ with $\xi_{n+1} \leq \frac{1}{(n+1)^{\frac{1}{t}}} \forall n \geq N_{*}$. By the triangle inequality, for each $m, n \in \mathbb{N}$ with $m>n$, we get

$$
\begin{aligned}
d_{K}\left(k_{n}, k_{m}\right) & \leq d_{K}\left(k_{n}, k_{n+1}\right)+d_{K}\left(k_{n+1}, k_{n+2}\right)+\cdots+d_{K}\left(k_{m-1}, k_{m}\right) \\
& \leq \sum_{j=n}^{\infty} \frac{1}{j^{\frac{1}{t}}} .
\end{aligned}
$$

Thus, $\lim _{n, m \rightarrow \infty} d_{K}\left(k_{n}, k_{m}\right)=0$, since $\sum_{j=1}^{\infty} \frac{1}{\frac{1}{t}}$ is convergent. Hence, we obtain a Cauchy sequence $\left\{k_{n}\right\}$ with $k_{n} R_{B} k_{n+1} \forall n \in \mathbb{W}$. As $\left(K, d_{K}\right)$ is complete in the sense of $R_{B}$, there is $k_{*} \in K$ with $k_{n} \rightarrow k_{*}$ and $k_{n} R_{B} k_{*}$ for each $n>m_{0}$ (for some $m_{0}$ ).

Suppose that $V$ is continuous with respect to $d_{K}$, then it is trivial that $V k_{*}=k_{*}$. 
Suppose that $F$ is continuous. Here, we claim $V k_{*}=k_{*}$. Assume in contrast that it is wrong. Then we have $d_{K}\left(V k_{n}, V k_{*}\right)>0 \forall n \geq m_{a}$. As $k_{n} R_{B} k_{*}$ for each $n>m_{b}=\max \left\{m_{a}, m_{0}\right\}$, by (2.11), we obtain

$$
\begin{aligned}
\Lambda+F\left(d_{K}\left(V k_{n}, V k_{*}\right)\right) \leq & F\left(\tau_{1} d_{K}\left(k_{n}, k_{*}\right)+\tau_{2} d_{K}\left(k_{n}, V k_{n}\right)\right. \\
& \left.+\tau_{3} d_{K}\left(k_{*}, V k_{*}\right)\right) \quad \forall n>m_{b} .
\end{aligned}
$$

As $\lim _{n \rightarrow \infty} d_{K}\left(k_{n}, k_{*}\right)=0, \lim _{n \rightarrow \infty} d_{K}\left(k_{n}, k_{n+1}\right)=0$, and $d_{K}\left(k_{*}, V k_{*}\right) \neq 0$, thus, there is $N_{0} \in$ $\mathbb{N}$ with

$$
\max \left\{d_{K}\left(k_{n}, k_{*}\right), d_{K}\left(k_{n}, k_{n+1}\right), d_{K}\left(k_{*}, V k_{*}\right)\right\}=d_{K}\left(k_{*}, V k_{*}\right) \quad \forall n \geq N_{0}
$$

By this fact and (2.19), we get

$$
\Lambda+F\left(d_{K}\left(k_{n+1}, V k_{*}\right)\right) \leq F\left(\left(\tau_{1}+\tau_{2}+\tau_{3}\right) d_{K}\left(k_{*}, V k_{*}\right)\right) \quad \forall n>\max \left\{m_{b}, N_{0}\right\}
$$

Let $n \rightarrow \infty$ in (2.20), by the continuity of $F$, we get

$$
\Lambda+F\left(d_{K}\left(k_{*}, V k_{*}\right)\right) \leq F\left(\left(\tau_{1}+\tau_{2}+\tau_{3}\right) d_{K}\left(k_{*}, V k_{*}\right)\right)
$$

which is not possible if $d_{K}\left(k_{*}, V k_{*}\right) \neq 0$, that is, a contradiction to our assumption. Hence, the claim is true, that is, $k_{*}=V k_{*}$. Consider $k=k_{*}$ in (2.12), we get $E\left(V k_{*}\right) \leq \lambda E\left(k_{*}\right)$, that is, $(1-\lambda) E\left(k_{*}\right) \leq 0$. Hence, $E\left(k_{*}\right)=0$.

\section{Consequences}

In this section, we list some results that are the consequences of the main results.

\subsection{Common fixed point result}

As a consequence of the above results, we get the following common fixed point result by defining $E(k)=d_{K}(k, S k) \forall k \in K$, where $S: K \rightarrow K$ is any map.

Corollary 3.1 Consider a metric space $\left(K, d_{K}\right)$ equipped with a binary relation $R_{B}$, and consider two maps $V, S: K \rightarrow K$ that satisfy the following two inequalities:

(1) either

$$
\Lambda+F\left(d_{K}(V k, V z)\right) \leq \tau_{1} F\left(d_{K}(k, z)\right)+\tau_{2} F\left(d_{K}(k, V k)\right)+\tau_{3} F\left(d_{K}(z, V z)\right)
$$

for each $k, z \in K$ with $k R_{B} z$ and

$$
\min \left\{d_{K}(V k, V z), d_{K}(k, V k), d_{K}(z, V z)\right\}>0
$$

where $F \in \Omega, \Lambda>0$ and $\tau_{1}, \tau_{2}, \tau_{3} \in(0,1)$ with $\tau_{1}+\tau_{2}+\tau_{3}=1$,

or

$$
\Lambda+F\left(d_{K}(V k, V z)\right) \leq F\left(\tau_{1} d_{K}(k, z)+\tau_{2} d_{K}(k, V k)+\tau_{3} d_{K}(z, V z)\right)
$$

for each $k, z \in K$ with $k R_{B} z$ and $V k \neq V z$, where $F \in \Omega, \Lambda>0$ and $\tau_{1}, \tau_{2}, \tau_{3} \in[0,1]$ with $\tau_{1}+\tau_{2}+\tau_{3}=1$. 
(2)

$$
d_{K}(V k, S V k) \leq \lambda d_{K}(k, S k) \quad \forall k \in K,
$$

where $\lambda \in[0,1)$. Also, consider that

(a) there exist $k_{0} \in K$ and $k_{1}=V k_{0}$ such that $k_{0} R_{B} k_{1}$;

(b) if $k, l \in K$ with $k R_{B} l$, then $V k R_{B} V l$, that is, $V$ is $R_{B}$-preserving;

(c) $\left(K, d_{K}\right)$ is complete in the sense of $R_{B}$;

(d) either $V$ is continuous or $F$ is continuous.

Then there exists a point $b \in K$ with $V b=b$ and $S b=b$.

\subsection{Result on a metric space equipped with graph}

Suppose that $G=\left(V_{E}, E_{D}\right)$ denotes a directed graph with a vertex set $V_{E}=K$ and an edge set $E_{D} \subset K \times K$ such that $E_{D}$ does not contain parallel edges but contains each loop, that is, $(k, k) \in E_{D} \forall k \in K$.

The following stated result is an explicit consequence of Theorem 2.2 and Theorem 2.4 by defining a binary relation $R_{B}$ on $K$ as $k R_{B} l$ if $(k, l) \in E_{D}$.

Corollary 3.2 Consider a metric space $\left(K, d_{K}\right)$ equipped with the graph $G$. Also, consider maps $V: K \rightarrow K$ and $E: K \rightarrow[0, \infty)$ that satisfy the following two inequalities:

(1) either

$$
\Lambda+F\left(d_{K}(V k, V z)\right) \leq \tau_{1} F\left(d_{K}(k, z)\right)+\tau_{2} F\left(d_{K}(k, V k)\right)+\tau_{3} F\left(d_{K}(z, V z)\right)
$$

for each $k, z \in K$ with $(k, z) \in E_{D}$ and

$$
\min \left\{d_{K}(V k, V z), d_{K}(k, V k), d_{K}(z, V z)\right\}>0,
$$

where $F \in \Omega, \Lambda>0$ and $\tau_{1}, \tau_{2}, \tau_{3} \in(0,1)$ with $\tau_{1}+\tau_{2}+\tau_{3}=1$,

or

$$
\Lambda+F\left(d_{K}(V k, V z)\right) \leq F\left(\tau_{1} d_{K}(k, z)+\tau_{2} d_{K}(k, V k)+\tau_{3} d_{K}(z, V z)\right)
$$

for each $k, z \in K$ with $(k, z) \in E_{D}$ and $V k \neq V z$, where $F \in \Omega, \Lambda>0$ and $\tau_{1}, \tau_{2}, \tau_{3} \in[0,1]$ with $\tau_{1}+\tau_{2}+\tau_{3}=1$.

(2)

$$
E(V k) \leq \lambda E(k) \quad \forall k \in K
$$

where $\lambda \in[0,1)$. Also, consider that

(a) there exist $k_{0} \in K$ and $k_{1}=V k_{0}$ such that $\left(k_{0}, k_{1}\right) \in E_{D}$;

(b) if $k, l \in K$ with $(k, l) \in E_{D}$, then $(V k, V l) \in E_{D}$, that is, $V$ is $E_{D}$-preserving;

(c) $\left(K, d_{K}\right)$ is complete in the sense of $G$, that is, for each sequence $\left\{k_{n}\right\}$ with

$\lim _{n, m \rightarrow \infty} d_{K}\left(k_{n}, k_{m}\right)=0$ and $\left(k_{n}, k_{n+1}\right) \in E_{D} \forall n \in \mathbb{N}$, we have $k_{b} \in K$ with $\lim _{n \rightarrow \infty} d_{K}\left(k_{n}, k_{b}\right)=0$ and $\left(k_{n}, k_{b}\right) \in E_{D} \forall n \geq n_{0}\left(\right.$ for some $\left.n_{0} \in \mathbb{N}\right) ;$

(d) either $V$ is continuous or $F$ is continuous.

Then there exists a point $b \in K$ with $V b=b$ and $E b=0$. 


\subsection{Result on a metric space with a function $\alpha$}

The result stated below has been obtained from Theorems 2.2 and 2.4 by taking a binary relation $R_{B}$ on $K$ as $k R_{B} l$ if $\alpha(k, l) \geq 1$, where $\alpha: K \times K \rightarrow[0, \infty)$ is a function.

Corollary 3.3 Consider a metric space $\left(K, d_{K}\right)$ and a function $\alpha: K \times K \rightarrow[0, \infty)$. Also, consider the maps $V: K \rightarrow K$ and $E: K \rightarrow[0, \infty)$ that satisfy the following two inequalities:

(1) either

$$
\Lambda+F\left(d_{K}(V k, V z)\right) \leq \tau_{1} F\left(d_{K}(k, z)\right)+\tau_{2} F\left(d_{K}(k, V k)\right)+\tau_{3} F\left(d_{K}(z, V z)\right)
$$

for each $k, z \in K$ with $\alpha(k, z) \geq 1$ and

$$
\min \left\{d_{K}(V k, V z), d_{K}(k, V k), d_{K}(z, V z)\right\}>0
$$

where $F \in \Omega, \Lambda>0$ and $\tau_{1}, \tau_{2}, \tau_{3} \in(0,1)$ with $\tau_{1}+\tau_{2}+\tau_{3}=1$,

or

$$
\Lambda+F\left(d_{K}(V k, V z)\right) \leq F\left(\tau_{1} d_{K}(k, z)+\tau_{2} d_{K}(k, V k)+\tau_{3} d_{K}(z, V z)\right)
$$

for each $k, z \in K$ with $\alpha(k, z) \geq 1$ and $V k \neq V z$, where $F \in \Omega, \Lambda>0$ and $\tau_{1}, \tau_{2}, \tau_{3} \in[0,1]$ with $\tau_{1}+\tau_{2}+\tau_{3}=1$.

(2)

$$
E(V k) \leq \lambda E(k) \quad \forall k \in K
$$

where $\lambda \in[0,1)$. Also, consider that

(a) there exist $k_{0} \in K$ and $k_{1}=V k_{0}$ such that $\alpha\left(k_{0}, k_{1}\right) \geq 1$;

(b) if $k, l \in K$ with $\alpha(k, l) \geq 1$, then $\alpha(V k, V l) \geq 1$, that is, $V$ is $\alpha$-preserving/admissible;

(c) $\left(K, d_{K}\right)$ is complete in the sense of $\alpha$, that is, for each sequence $\left\{k_{n}\right\}$ with $\lim _{n, m \rightarrow \infty} d_{K}\left(k_{n}, k_{m}\right)=0$ and $\alpha\left(k_{n}, k_{n+1}\right) \geq 1 \forall n \in \mathbb{N}$, we have $k_{b} \in K$ with $\lim _{n \rightarrow \infty} d_{K}\left(k_{n}, k_{b}\right)=0$ and $\alpha\left(k_{n}, k_{b}\right) \geq 1 \forall n \geq n_{0}\left(\right.$ for some $\left.n_{0} \in \mathbb{N}\right)$;

(d) either $V$ is continuous, or $F$ is continuous.

Then there exists a point $b \in K$ with $V b=b$ and $E b=0$.

\section{Application and examples}

Consider an integral equation

$$
k(w)=h(w)+\mu \int_{0}^{f(w)} M(w, q, k(q)) d q, \quad w \in I_{N}=[0, \infty),
$$

where $\mu$ is constant, $h: I_{N} \rightarrow \mathbb{R}, f: I_{N} \rightarrow \mathbb{R}^{+}=[0, \infty)$, and $M: I_{N} \times I_{N} \times \mathbb{R} \rightarrow \mathbb{R}$ are continuous functions.

Also, consider $K=C\left(I_{N}, \mathbb{R}\right)$ to be the set that contains all real-valued continuous functions with the domain set $I_{N}$ and $d_{K}(k, l)=\max _{w \in I_{N}}|k(w)-l(w)|=\|k-l\|$. 
Theorem 4.1 Consider $K=C\left(I_{N}, \mathbb{R}\right)$ and consider an operator $V: K \rightarrow K$ defined by

$$
V k(w)=h(w)+\mu \int_{0}^{f(w)} M(w, q, k(q)) d q, \quad w \in I_{N}=[0, \infty)
$$

where $\mu$ is constant, $h: I_{N} \rightarrow \mathbb{R}, f: I_{N} \rightarrow \mathbb{R}^{+}=[0, \infty)$, and $M: I_{N} \times I_{N} \times \mathbb{R} \rightarrow \mathbb{R}$ are continuous functions. Also, assume that there are $\Lambda>0$ and $\tau_{1}, \tau_{2}, \tau_{3} \in(0,1)$ with $\tau_{1}+\tau_{2}+$ $\tau_{3}=1$ satisfying

$$
|M(w, q, k(q))-M(w, q, l(q))| \leq \frac{|k(q)-l(q)|}{\left[\Lambda \sqrt{\|k-l\|}+\tau_{1}+\tau_{2} \sqrt{\frac{\|k-l\|}{\|k-V k\|}}+\tau_{3} \sqrt{\frac{\|k-l\|}{\|l-V l\|}}\right]^{2}}
$$

for all $w, q \in I_{N}$ and for each $k, l \in K$ with $\min \{\|V k-V l\|,\|k-V k\|,\|l-V l\|\}>0$; moreover,

$$
\sup _{w \in I_{N}} \int_{0}^{f(w)} d q \leq \frac{1}{|\mu|}
$$

Then (4.1) possesses a solution.

Proof By (4.2) and (4.3), for each $k, l \in K$ with $\min \{\|V k-V l\|,\|k-V k\|,\|l-V l\|\}>0$, we reach

$$
\begin{aligned}
|V k(w)-V l(w)| & \leq|\mu| \int_{0}^{f(w)}|M(w, q, k(q))-M(w, q, l(q))| d q \\
& \leq|\mu| \int_{0}^{f(w)} \frac{|k(q)-l(q)|}{\left[\Lambda \sqrt{\|k-l\|}+\tau_{1}+\tau_{2} \sqrt{\frac{\|k k-l\|}{\|k-V k\|}}+\tau_{3} \sqrt{\frac{\|k-l\|}{\|l-V l\|}}\right]^{2}} d q \\
& \leq \frac{\|k-l\|}{\left[\Lambda \sqrt{\|k-l\|}+\tau_{1}+\tau_{2} \sqrt{\frac{\|k-l\|}{\|k-V k\|}}+\tau_{3} \sqrt{\frac{\|k-l\|}{\|l-V l\|}}\right]^{2}}|\mu| \int_{0}^{f(w)} d q \\
& \leq \frac{\|k-l\|}{\left[\Lambda \sqrt{\|k-l\|}+\tau_{1}+\tau_{2} \sqrt{\frac{\|k-l\|}{\|k-V k\|}}+\tau_{3} \sqrt{\frac{\|k-l\|}{\|l-V l\|}}\right]^{2}} \quad \forall w \in I_{N} .
\end{aligned}
$$

This gives

$$
\|V k-V l\| \leq \frac{\|k-l\|}{\left[\Lambda \sqrt{\|k-l\|}+\tau_{1}+\tau_{2} \sqrt{\frac{\|k-l\|}{\|k-V k\|}}+\tau_{3} \sqrt{\frac{\|k-l\|}{\|l-V l\|}}\right]^{2}} .
$$

This implies

$$
\begin{aligned}
\frac{1}{\sqrt{\|V k-V l\|}} \geq & \frac{\left[\Lambda \sqrt{\|k-l\|}+\tau_{1}+\tau_{2} \sqrt{\frac{\|k-l\|}{\|k-V k\|}}+\tau_{3} \sqrt{\frac{\|k-l\|}{\|l-V l\|}}\right]}{\sqrt{\|k-l\|}} \\
& =\Lambda+\frac{\tau_{1}}{\sqrt{\|k-l\|}}+\frac{\tau_{2}}{\sqrt{\|k-V k\|}}+\frac{\tau_{3}}{\sqrt{\|l-V l\|}}
\end{aligned}
$$

So, we get

$$
\Lambda-\frac{1}{\sqrt{\|V k-V l\|}} \leq \frac{-\tau_{1}}{\sqrt{\|k-l\|}}+\frac{-\tau_{2}}{\sqrt{\|k-V k\|}}+\frac{-\tau_{3}}{\sqrt{\|l-V l\|}}
$$


Hence,

$$
\Lambda+F\left(d_{K}(V k, V l)\right) \leq \tau_{1} F\left(d_{K}(k, l)\right)+\tau_{2} F\left(d_{K}(k, V k)\right)+\tau_{3} F\left(d_{K}(l, V l)\right)
$$

for each $k, l \in K$ with $\min \left\{d_{K}(V k, V l), d_{K}(k, V k), d_{K}(l, V l)\right\}>0$, where $F(w)=\frac{-1}{\sqrt{w}}$. By defining a binary relation $R_{B}$ on $K$ as $k R_{B} l$ if $(k, l) \in K \times K$ and $E(k)=0$ for each $k \in K$, we see that the axioms of Theorem 2.2 become true. Hence, we say that $V$ contains a fixed point in $K$, that is, (4.1) possesses a solution.

\section{Illustration by examples}

Example 4.2 Consider $K=\mathbb{R}$ and define

$$
d_{K}(k, l)= \begin{cases}0, & k=l, \\ \max \{|k|,|l|\}+1, & \text { otherwise }\end{cases}
$$

Define a binary relation $R_{B}$ on $K$ by $k R_{B} l$ if $k, l \in[0,1]$. Define $V: K \rightarrow K$ and $E: K \rightarrow$ $[0, \infty)$ by

$$
V(k)= \begin{cases}2 k, & K \backslash[0,1], \\ 0, & k \in[0,1 / 3], \\ \frac{1}{9}, & k \in(1 / 3,1]\end{cases}
$$

and

$$
E(k)= \begin{cases}k, & k \in[0,1] \\ \frac{1}{|k|}, & \text { otherwise }\end{cases}
$$

In this example, one can see that the axioms of Theorem 2.2 are valid by taking $F(k)=\ln (k)$ and $\Lambda=0.001$. Thus, there exists a point $b \in K$ such that $V b=b$ and $E b=0$.

Remark 4.3 ([7, Theorem 2]) is not applicable to the above defined $d_{K}$ and $V$.

Example 4.4 Consider $K=[0,2]$ and define $d_{K}(k, l)=|k-l| \forall k, l \in K$. Define a binary relation $R_{B}$ on $K$ by $k R_{B} l$ if $k, l \in\{0,1 / 3,2 / 3,1,2\}$. Define $V: K \rightarrow K$ and $E: K \rightarrow[0, \infty)$ by

$$
V(k)=\frac{\lceil k-0.9\rceil}{3} \quad \forall k \in K
$$

and

$$
E(k)=\lfloor k\rfloor \quad \forall k \in K .
$$

One can verify that the axioms of Theorem 2.4 are valid by taking $F(k)=\ln (k)$ and $\Lambda=$ 0.0001 , thus there exists a point $b \in K$ such that $V b=b$ and $E b=0$. 
Example 4.5 Consider $K=[0,2]$ and define $d_{K}(k, l)=|k-l| \forall k, l \in K$. Define a binary relation $R_{B}$ on $K$ by $k R_{B} l$ if $k, l \in\{0,1 / 3,2 / 3,1,2\}$. Define $V: K \rightarrow K$ and $E: K \rightarrow[0, \infty)$ by

$$
V(k)= \begin{cases}\lceil k-0.9\rceil & k \in\{0,1 / 3,2 / 3,1,2\}, \\ \lceil k-0.9\rceil, & \text { otherwise }\end{cases}
$$

and

$$
E(k)=0 \quad \forall k \in K .
$$

One can verify that the axioms of Theorem 2.4 are valid by taking $F(k)=\ln (k)$ and $\Lambda=$ 0.0001 , thus there exists a point $b \in K$ such that $V b=b$ and $E b=0$.

Remark 4.6 Note that for the function $V$ defined in the last example inequality (2.11) is valid only for $k, l \in K$ with $k R_{B} l$.

\section{Conclusion}

The notions of interpolative $F$-contractions with shrink map and $F$-contractions with shrink map have been defined and used to study the existence of $E$-fixed points on a metric space endowed with a binary relation. As an application and consequence of the main results, we have obtained a common fixed point result, an $E$-fixed point result on a metric space with graph, and an existence theorem for a solution of integral equations.

\section{Acknowledgements}

This work was supported by the Deanship of Scientific Research (DSR), King Abdulaziz University, Jeddah, Saudi Arabia, under grant number KEP-5-130-42. The authors, therefore, gratefully acknowledge the DSR for technical and financial support.

\section{Funding}

The Deanship of Scientific Research (DSR) at King Abdulaziz University, Jeddah, Saudi Arabia, has funded this project under grant number KEP-5-130-42.

\section{Availability of data and materials}

The data used to support the findings of this study are available from the corresponding author upon request.

\section{Competing interests}

The authors declare that they have no competing interests.

Authors' contributions

Both authors have contributed equally in writing this article. Both authors have read and approved the final manuscript.

\section{Author details}

'Department of Mathematics, King Abdulaziz University, P.O. Box 80203, Jeddah 21589, Saudi Arabia. ${ }^{2}$ Department of Mathematics, COMSATS University Islamabad, Attock Campus, Attock, Pakistan.

\section{Publisher's Note}

Springer Nature remains neutral with regard to jurisdictional claims in published maps and institutional affiliations.

Received: 16 March 2021 Accepted: 30 May 2021 Published online: 09 June 2021

\section{References}

1. Karapınar, E.: Revisiting the Kannan type contractions via interpolation. Adv. Theory Nonlinear Anal. Appl. 2, 85-87 (2018)

2. Karapınar, E., Agarwal, R.P., Aydi, H.: Interpolative Reich-Rus-Ciric type contractions on partial metric spaces. Mathematics 6(11), 256 (2018)

3. Gaba, Y.U., Karapınar, E.: A new approach to the interpolative contractions. Axioms 8(4), 110 (2019)

4. Karapınar, E., Algahtani, O., Aydi, H.: On interpolative Hardy-Rogers type contractions. Symmetry 11, 8 (2018) 
5. Aydi, H., Chen, C.M., Karapınar, E.: Interpolative Ciric-Reich-Rus type contractions via the Branciari distance. Mathematics 7(1), 84 (2019)

6. Karapınar, E.: Revisiting simulation functions via interpolative contractions. Appl. Anal. Discrete Math. 13(3), 859-870 (2019)

7. Mohammadi, B., Parvaneh, V., Aydi, H.: On extended interpolative Ciric-Reich-Rus type F-contractions and an application. J. Inequal. Appl. 2019, 290 (2019)

8. Wardowski, D.: Fixed points of a new type of contractive mappings in complete metric spaces. Fixed Point Theory Appl. 2012, 94 (2012)

9. Altun, l., Tasdemir, A.: On best proximity points of interpolative proximal contractions. Quaest. Math. (2020). https://doi.org/10.2989/16073606.2020.1785576

10. Debnath, P., de La Sen, M.: Fixed-points of interpolative Ciric-Reich-Rus-type contractions in b-metric spaces. Symmetry 12, 12 (2020)

11. Debnath, P., de La Sen, M.: Set-valued interpolative Hardy-Rogers and set-valued Reich-Rus-Ciric-type contractions in b-metric spaces. Mathematics 7(9), 849 (2019)

12. Ali, M.U., Alansari, M.: New generalizations of set-valued interpolative Hardy-Rogers type contractions in b-metric spaces. J. Funct. Spaces 2021, 6641342 (2021)

13. Karapınar, E., Aydi, H., Mitrovic, Z.D.: On interpolative Boyd-Wong and Matkowski type contractions. TWMS J. Pure Appl. Math. 11, 204-212 (2020)

14. Aydi, H., Karapinar, E., Roldán López de Hierro, A.F.: $\omega$-Interpolative Ciric-Reich-Rus-type contractions. Mathematics 7 , 57 (2019)

15. Ameer, E., Aydi, H., Arshad, M., Alsamir, H., Noorani, M.S.: Hybrid multivalued type contraction mappings in $\alpha$-K-complete partial b-metric spaces and applications. Symmetry 11, 86 (2019)

16. Parvaneh, V., Haddadi, M.R., Aydi, H.: On best proximity point results for some type of mappings. J. Funct. Spaces 2020, 6298138 (2020)

17. Aydi, H., Lakzian, H., Mitrović, Z.D., Radenović, S.: Best proximity points of MT-cyclic contractions with property UC. Numer. Funct. Anal. Optim. 41, 871-882 (2020)

18. Karapınar, E., Fulga, A., Agarwal, R.P.: A survey: F-contractions with related fixed point results. J. Fixed Point Theory Appl. 22, 1-58 (2020)

19. Patle, P., Patel, D., Aydi, H., Radenović, S.: On H+ type multivalued contractions and applications in symmetric and probabilistic spaces. Mathematics 7, 144 (2019)

20. Karapinar, E., Czerwik, S., Aydi, H.: $(\alpha, \psi)$-Meir-Keeler contraction mappings in generalized b-metric spaces. J. Funct. Spaces 2018, $3264620(2018)$

21. Ali, M.U., Pitea, A.: Existence theorem for integral inclusions by a fixed point theorem for multivalued implicit-type contractive mappings. Nonlinear Anal., Model. Control 26, 334-348 (2021)

22. Ali, M.U., Ionescu, C., Stanciu, M.: On ( $\phi, \varphi)$-best proximity points for proximal type contraction mappings. UPB Sci. Bull., Ser. A 83, 15-26 (2021)

23. Jleli, M., Samet, B., Vetro, C.: Fixed point theory in partial metric spaces via $\phi$-fixed point's concept in metric spaces. J. Inequal. Appl. 2014, 426 (2014)

\section{Submit your manuscript to a SpringerOpen ${ }^{\circ}$ journal and benefit from:}

- Convenient online submission

- Rigorous peer review

- Open access: articles freely available online

- High visibility within the field

- Retaining the copyright to your article

Submit your next manuscript at $\boldsymbol{\nabla}$ springeropen.com 